\title{
A Static Bioimpedance and Dynamic Acoustic Myography Preliminary Assessment of Low Frequency Therapeutic Ultrasound Treatment of the Shoulder Muscle Trapezius: An Equine Study
}

\author{
Diane lsbell1, Sebastian Laguna ${ }^{2}$, Adrian Harrison ${ }^{3 *}$ \\ ${ }^{1}$ Equine Vet Practice, Livermore, CA, USA \\ ${ }^{2}$ University of La Salle Veterinary Sciences, Bogota, Colombia \\ ${ }^{3}$ Biomechanics \& Rehabilitation, Section for Pathobiological Sciences, Faculty of Health \& Medical Sciences, Copenhagen \\ University, Copenhagen, Denmark \\ Email:^adh@sund.ku.dk
}

How to cite this paper: 1sbell, D., Laguna, S. and Harrison, A. (2021) A Static Bioimpedance and Dynamic Acoustic Myography Preliminary Assessment of Low Frequency Therapeutic Ultrasound Treatment of the Shoulder Muscle Trapezius: An Equine Study. Open Journal of Veterinary Medicine, 11, 125-135.

https://doi.org/10.4236/ojvm.2021.114008

Received: March 16, 2021

Accepted: April 23, 2021

Published: April 26, 2021

Copyright $\odot 2021$ by author(s) and Scientific Research Publishing Inc. This work is licensed under the Creative Commons Attribution International License (CC BY 4.0).

http://creativecommons.org/licenses/by/4.0/

\section{(c) (i) Open Access}

\begin{abstract}
Background: In horses, therapeutic ultrasound has been shown to be beneficial for suspensory ligament injuries, and more generally to stimulate tissue repair and reduce localized pain. However, it has yet to be examined in relation to soft tissue injuries sustained during competition or in connection with rider/saddle interactions. Aim: The aim of the present study was to examine the efficacy of topical low-frequency therapeutic ultrasound on the shoulder muscles of injured horses. Methods: Muscle mass and cellular health was assessed for $M$. trapezius using non-invasive multi-frequency bioimpedance, and dynamic improvements in M. trapezius and M. latissimus dorsi were examined using acoustic myography (AMG). A total of 8 injured horses (painful to palpation and atrophied) were treated using an EQ Pro Therapy unit, following a set protocol that comprised treatment every other day for 10 treatments. Pre-treatment values were then compared with post-treatment measurements ( 1 \& 6 months later). Results: The mfBIA results for $M$. trapezius showed a significant improvement $(\mathrm{P}<0.05)$ of EQ Pro Therapy treatment for the membrane capacitance $(\mathrm{Mc})$ and phase angle $(\mathrm{PA})$ parameters when comparing the Pre vs Post 1 and Post 1 vs Post 2 measurements. Similar improvements were seen for the AMG measurements for M. trapezius and $M$. latissimus dorsi where statistically significant improvements in the muscle efficiency (E-score) and temporal summation (T-score) as well as the spatial summation (S-score) and mean of the ST-scores were noted $(\mathrm{P}<0.05$ to $\mathrm{P}<$
\end{abstract}


0.01). Conclusion: The present findings suggest that EQ Pro Therapy treatment is efficacious in terms of equine cases involving soft tissue shoulder muscle injuries (painful to palpation and atrophied). The main improvements noted were not only increased muscle mass and cellular health but also improved force production, better coordination, and lateral balance, which persisted for at least six months after treatment.

\section{Keywords}

Horse, Skeletal Muscle, Acoustic Myography, Low Frequency Ultrasound

\section{Introduction}

It has been reported that injuries to the skeletal and muscular systems of horses remain the primary reason for them being euthanized [1]. Indeed, a Swedish study of 8281 horses, assessed over a period of 22 years, revealed that $11 \%$ had flexion test issues relating to the forelimb region, and of those cases with a positive palpatory orthopaedic examination, $57 \%$ were identified as relating to the shoulder muscles [1]. More recently it has been reported that the degree of loading to the back of horses affects their movement mechanics [2]. Wilk and colleagues [2] used thermal imaging to show that loading significantly increased the superficial temperature of the front of trunk (shoulder muscles) region compared with values at rest, a region that not only bears a proportion of the rider's weight but is also important for saddle contact and fit. Of course, a saddle should not only ensure a rider's safety and comfort, it should also facilitate an even distribution of pressure to the horse's shoulders and back [3]. Thus, a saddle that is a poor fit for a horse causes instability in the rhythmic motion of the horse's back as well as its range of motion [4], ultimately translating into health and lameness issues in the back and limbs [2].

Therapeutic ultrasound is a commonly used treatment for a variety of conditions, ranging from pain management, oedema, muscle spasms, and tissue repair [5]. Recently, it was shown that low-frequency therapeutic ultrasound $(38 \mathrm{kHz})$, was effective as a form of treatment for horses with suspensory ligament injury [6]. Thus, since this mode of treatment is non-invasive and comprises simple massaging of an injured area in combination with a traditional ultrasound gel, we were interested to establish whether it could be used as part of a rehabilitation program for horses with the muscular shoulder injury.

The present study has therefore assessed any benefits of topical low-frequency therapeutic ultrasound on the shoulder muscles of horses, as assessed by the static measurement of multi-frequency bioimpedance (mfBIA) and the dynamic measurement of acoustic myography (AMG) for muscle function monitoring [7] [8]. The hypothesis was that improvements in both mfBIA and AMG parameters, indicative of cellular health and muscle function, would occur with lowfrequency therapeutic ultrasound treatment in the shoulder muscle of injured 
horses.

\section{Materials \& Methods}

\subsection{Ethics}

The study was carried out in accordance with the Helsinki Declaration. The owners of the horses used, gave their informed consent prior to the start of this study. In addition, this study was entirely non-invasive in its nature, and full ethical approval was gained from Copenhagen University Department of Pathobiological Sciences, Faculty of Health \& Medical Sciences before the start of the trial.

\subsection{Subjects}

Ten subjects were recruited for the present study (Isbell DVM; Livermore, USA). Of the 10 that started the trial, two horses died of unrelated causes before the post treatment measurements could be taken ( 1 horse died of colic, 1 horse was euthanized after a fence injury that destroyed the hock ligaments), so a final total of 8 horses were included in the data set. The average age of the horses in this study was 14.3 years, which included 3 Thoroughbreds, 1 Arabian and 4 Warmbloods. Of the 8 horses, seven of them were geldings. The inclusion criteria were the following; horses that were being rested, had a large turn-out area so that exercise would not be a factor in muscle change, and had muscles that were both mildly painful to palpation and atrophied. All the horses were evaluated through manual palpation of the muscle region, as well as a complete physical and lameness evaluation by a qualified and experienced veterinarian (DI) and any horses exhibiting severe pain responses were excluded from the study. A severe pain response was assessed in accordance with the published grimace scale [9].

\subsection{Experimental Protocol}

The protocol adopted in this study was to identify muscles that are often painful to palpation and atrophied. These are often muscles at the shoulders, or along the back that can be injured during exercise, perhaps as a result of an ill-fitting saddle. The plan was then to measure the state of the trapezius muscle both statically and dynamically before treatment, and at two set times post treatment (Post 1 and Post 2). Post 1 measurements were undertaken 1 month after the start of treatment, and Post 2 measurements were undertaken 6 months after treatment start. In between these two, post treatment time points the horses were not actively exercised but were simply allowed out to pasture on a daily basis.

\section{4. $\mathrm{mfBIA}$}

Multi-frequency Bioimpedance (mfBIA; ImpediVET BIS 1, Pinkenba, Australia) was used to assess the shoulder muscle trapezius (cervical) in a static state. In brief, this technique relies on the application of an alternating low-level current which is sent through the muscle of interest, whilst the voltage is measured 
across the same site. Using Ohm's law it is then possible to measure the cellular mass and health of the tissue and with the aid of different frequencies, to assess not only the intracellular resistance ( $\mathrm{Ri}$ ) but also the centre frequency (fc), membrane capacitance $(\mathrm{Mc})$ and overall level of tissue health using the phase angle (PA). For some reference equine muscle values and more details about mfBIA see Harrison and colleagues [7].

\subsection{Acoustic Myography}

Acoustic myography (AMG) is a biomechanical method that measures the pressure waves generated by active muscle contractions [8]. AMG recordings were carried out for the muscles trapezius and latissimus dorsi (the later selected for its role in shoulder flexion and limb retraction) with a CURO unit and CURO sensors (CURO-Diagnostics ApS, Denmark; formerly MyoDynamikApS) and followed in real time on an iPad Air (Apple Inc, Cupertino, CA, USA) via the App "CURO Equine" and a specialized data recording system. This allowed us to see the actual recordings while the horses were physically active. We used $20 \mathrm{~mm}$ sensors with a frequency recording range of $0.5-20 \pm 0.5 \mathrm{kHz}$, and the sampling rate was $4 \mathrm{kHz}$. Recorded data was stored to the CURO Unit and after completion of measurements transferred to the CURO software (https://app.myodynamik.com).

In brief, ultrasound-gel coated sensors were placed centrally on the muscles of interest, connecting cables were run from the sensors and connected to the CURO unit which was placed in a pouch mounted on a surcingle fastened to the horses. The sensors and the wires from the sensors were secured with a flexible adhesive bandage (Snøgg AS; Norway).

The AMG signal comprises three components: 1) efficiency (E-score) 2) temporal fibre recruitment (T-score) and 3) spatial fibre recruitment (S-score), calculated using the CURO software [8] [10].

As an example, the $\mathrm{S}$-score was determined as the signal amplitude in relation to a full $6 \mathrm{~dB}$ signal (measured as approx. $1 \mathrm{~V}$ ). For more intuitive assessment of optimal muscle function, a scale of 0 - 10 was adopted, where 0 was considered as $0 \%$ optimal and 10 was considered $100 \%$ optimal. To calculate the score, the measured $\mathrm{mV}$ amplitude was subtracted from the maximal $\mathrm{mV}$ amplitude that could be accurately detected. The difference was then divided by the maximal amplitude and multiplied by 10 to yield a $0-10$ scoring system. By way of an example, an S-score of 8 represents a signal with a very small amplitude (approx. $0.3 \mathrm{~V}$ ), whereas an S-score of 1 represents a relatively large amplitude signal (approx. $0.7 \mathrm{~V}$ ). The T- and E-score were calculated in a similar way to their full signal to give a score scale from $0-10$, where 0 is full activity and 10 is no activity. For further details see Harrison [8].

\subsection{Exercise}

For the dynamic assessment of muscle function involving AMG, horses were 
walked in a straight line for approx. 2 mins thereafter they were trotted, also in a straight line, for a further approx. 2 mins. The exercise took place on an even, firm surface, and was the same for all the horses measured in this study.

\subsection{Low Frequency Ultrasound}

The device used for low-frequency ultrasound therapeutic treatments was an EQ Pro (EQ Veterinary, Daytona Beach, Florida USA; http://www.eqveterinary.com). This device produces a low-frequency therapeutic ultrasound, which operates at $38 \mathrm{kHz}( \pm 2 \mathrm{kHz})$. The unit comes with six different color-coded transducers, featuring continuous and pulsed emission and with different transducer shapes to allow treatment of different musculoskeletal pathologies (for details see: http://www.eqveterinary.com) [6].

\subsection{Treatment}

The two advantages of using low-frequency therapeutic US versus high frequency US are increased intensity of induced acoustic effects and depth of penetration. A hand-held transducer of the type "RED" was used for the treatment regions. The "RED" transducer features a flat head and continuous emission and generates acoustic effects and thermal effects: it is ideal to treat large and flat areas and when heat is the desired effect (see Figure 1).

EQ Pro therapy does not require horses to be sedated or treatment areas to be shaved, only that they are cleaned with water and left wet so as to facilitate the EQ Pro transduction. US-specific gel was applied to both the contact surface of the EQ Pro transducer and directly to the treatment area. This was done so as to further facilitate penetration, but also to assist the transducer head to smoothly glide over the treatment area. Subsequent applications of US gel were made when it was felt that insufficient gel was present at the treatment site. The power output was set to $80 \%-100 \%$ and a treatment time of 10 minutes was used, with the transducer being constantly moved over the hair and skin surface, equivalent

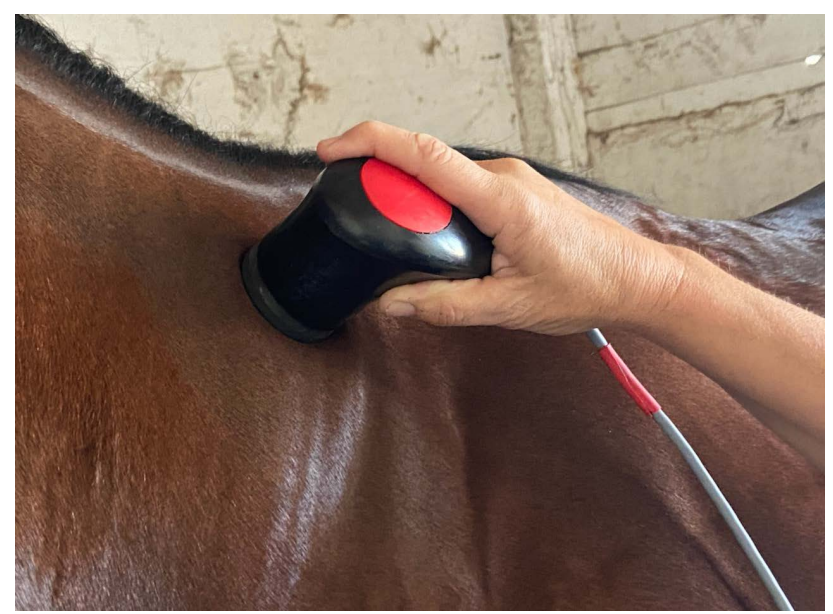

Figure 1. A photograph taken during treatment of the hand-held transducer of the type "RED" in place on the cervical region of the shoulder muscle trapezius. 
to an area of approx. $21 \times 27 \mathrm{~cm}$. Treatment was performed by massaging the selected area of the horse applying slight pressure and maintaining the US transducer in direct contact with horse's gel enriched hair. This treatment protocol was repeated every other day for 10 treatments. For details concerning the heads and treatment protocols see (http://www.eqveterinary.com) [6].

\subsection{Statistics}

All statistics were performed using GraphPad InStat 3 for Mac (Version 3.0b, 2003; Graph- Pad Inc., La Jolla, CA). Data were initially tested for normal distribution and equal variance, and then subsequently analyzed using a t-test. Differences between means with a $\mathrm{P}$ value $>0.05$ were considered non-significant. Values are presented as the mean \pm the standard error of the mean.

\section{Results}

$m f B I A$. The mfBIA data for $M$. trapezius can be found in Figure 2. Whilst there was a tendency for the Ri parameter to decline over time, this was not significant due to a large variation in the data set. However, both the right-hand side $M$. trapezius $\mathrm{Mc}$ and $\mathrm{PA}$ parameters were found to be significantly improved $(\mathrm{P}<$ 0.05) when comparing the Pre vs Post 1 and Post1 vs Post 2 values. No significant changes in the mfBIA parameters were noted for the left-hand side of this muscle group although similar tendencies to those for the right-hand side were seen.
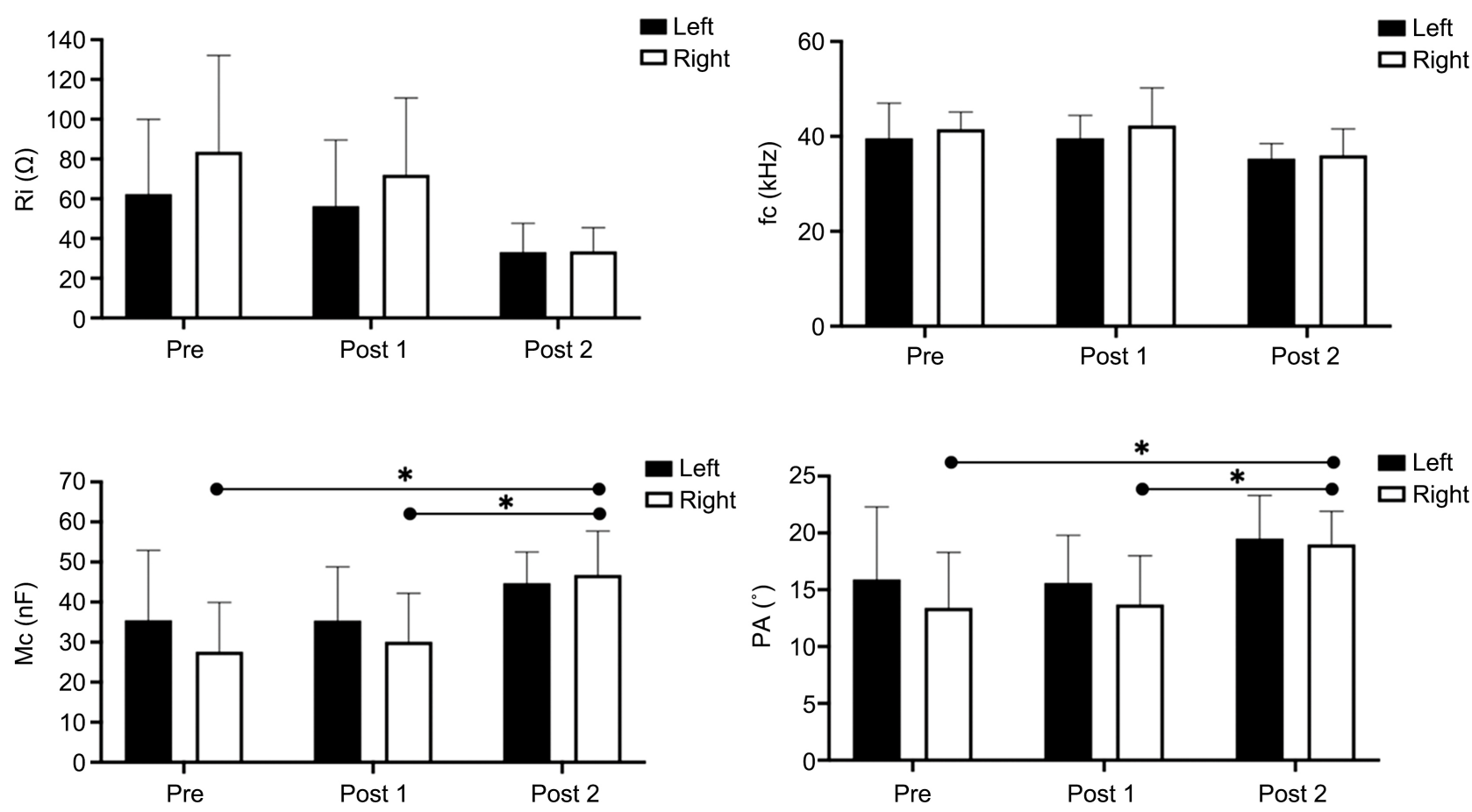

Figure 2. mfBIA data for the shoulder muscle trapezius taken prior to the start of treatment, after treatment (Post 1) and after treatment (Post 2) for both the left- and right-hand sides of the horses. Values are presented as the mean \pm SD of $n=8$. Statistically significant differences between means are denoted by a line and $\mathrm{a}^{*}=\mathrm{P}<0.05$. 
$A M G$. The AMG data for M. trapezius and M. latissimus dorsi can be found in Table 1, Table 2 and Table 3, Table 4. For M. trapezius at the walk, there was a statistically significant improvement in the $\mathrm{E}$ and T-scores for the left-side, and for the E, S and ST-scores for the right-side between the Pre and Post 2 measurements $(\mathrm{P}<0.05$ to $\mathrm{P}<0.01)$.

Table 1. M. trapezius AMG E, S and T-scores as well as the mean ST-score at the walk for the left- and right-sides of the horses measured in this study. ${ }^{*}=\mathrm{P}<0.05$ and ${ }^{* *}=\mathrm{P}<0.01$.

\begin{tabular}{|c|c|c|c|c|c|c|c|c|}
\hline \multirow{2}{*}{ Walk } & \multicolumn{4}{|c|}{ Left side } & \multicolumn{4}{|c|}{ Right side } \\
\hline & $\mathrm{E}$ & $S$ & $\mathrm{~T}$ & ST & $\mathrm{E}$ & S & $\mathrm{T}$ & ST \\
\hline Pre & $1.7 \pm 1.1$ & $6.8 \pm 1.7$ & $5.2 \pm 2.1$ & $6.0 \pm 1.8$ & $2.7 \pm 2.5$ & $6.7 \pm 2.5$ & $4.8 \pm 2.3$ & $5.7 \pm 1.6$ \\
\hline Post 1 & $3.9 \pm 3.4$ & $6.4 \pm 2.9$ & $7.1 \pm 1.2$ & $6.7 \pm 1.5$ & $5.0 \pm 3.5$ & $7.1 \pm 1.5$ & $6.8 \pm 1.9$ & $6.9 \pm 1.4$ \\
\hline Post 2 & $5.6 \pm 3.2^{\star *}$ & $7.3 \pm 1.5$ & $7.3 \pm 2.2^{\star}$ & $7.3 \pm 1.7$ & $5.9 \pm 3.3^{*}$ & $7.3 \pm 2.0$ & $7.0 \pm 1.8^{*}$ & $7.2 \pm 1.6^{*}$ \\
\hline
\end{tabular}

Table 2. M. trapezius AMG E, S and T-scores as well as the mean ST-score at the trot for the left- and right-sides of the horses measured in this study. ${ }^{* *}=\mathrm{P}<0.01$.

\begin{tabular}{|c|c|c|c|c|c|c|c|c|}
\hline \multirow{2}{*}{ Trot } & \multicolumn{4}{|c|}{ Left side } & \multicolumn{4}{|c|}{ Right side } \\
\hline & E & S & $\mathrm{T}$ & ST & $\mathrm{E}$ & S & $\mathrm{T}$ & ST \\
\hline Pre & $0.2 \pm 0.1$ & $2.6 \pm 1.7$ & $6.1 \pm 2.5$ & $4.4 \pm 1.8$ & $0.8 \pm 0.8$ & $4.1 \pm 1.8$ & $6.8 \pm 2.1$ & $5.5 \pm 1.2$ \\
\hline Post 1 & $1.1 \pm 1.6$ & $3.5 \pm 3.5$ & $7.4 \pm 1.1$ & $5.5 \pm 1.8$ & $0.9 \pm 2.8$ & $4.3 \pm 2.9$ & $7.8 \pm 1.7$ & $6.0 \pm 1.3$ \\
\hline Post 2 & $1.8 \pm 1.8^{\star *}$ & $3.3 \pm 2.9$ & $7.7 \pm 0.5$ & $5.5 \pm 1.6$ & $1.4 \pm 2.1$ & $2.9 \pm 3.6$ & $7.6 \pm 0.9$ & $5.6 \pm 2.1$ \\
\hline
\end{tabular}

Table 3. M. latissimus dorsi AMG E, S and T-scores as well as the mean ST-score at the walk for the left- and right-sides of the horses measured in this study. ${ }^{*}=\mathrm{P}<0.05$.

\begin{tabular}{|c|c|c|c|c|c|c|c|c|}
\hline \multirow{2}{*}{ Walk } & \multicolumn{4}{|c|}{ Left side } & \multicolumn{4}{|c|}{ Right side } \\
\hline & $\mathrm{E}$ & S & $\mathrm{T}$ & ST & $\mathrm{E}$ & S & $\mathrm{T}$ & ST \\
\hline Pre & $6.0 \pm 3.2$ & $8.6 \pm 0.7$ & $6.7 \pm 1.7$ & $7.6 \pm 1.2$ & $6.8 \pm 2.7$ & $9.0 \pm 0.2$ & $8.3 \pm 1.1$ & $8.7 \pm 0.6$ \\
\hline Post 1 & $6.9 \pm 2.2^{*}$ & $8.7 \pm 0.8$ & $8.4 \pm 1.1$ & $8.6 \pm 0.8$ & $8.3 \pm 1.6^{*}$ & $8.9 \pm 0.8$ & $8.7 \pm 1.5^{\star}$ & $8.7 \pm 0.7$ \\
\hline Post 2 & $4.7 \pm 3.6$ & $7.7 \pm 1.8$ & $7.6 \pm 1.5$ & $7.7 \pm 1.5$ & $5.7 \pm 3.6^{*}$ & $7.6 \pm 1.8^{\star}$ & $6.9 \pm 2.8^{\star}$ & $7.2 \pm 2.0^{*}$ \\
\hline
\end{tabular}

Table 4. M. latissimus dorsi AMG E, S and T-scores as well as the mean ST-score at the trot for the left- and right-sides of the horses measured in this study. ${ }^{*}=\mathrm{P}<0.05$ and ${ }^{* *}=\mathrm{P}<0.01$.

\begin{tabular}{|c|c|c|c|c|c|c|c|c|}
\hline \multirow{2}{*}{ Trot } & \multicolumn{4}{|c|}{ Left side } & \multicolumn{4}{|c|}{ Right side } \\
\hline & E & S & $\mathrm{T}$ & ST & $\mathrm{E}$ & S & $\mathrm{T}$ & ST \\
\hline Pre & $1.1 \pm 0.8$ & $5.4 \pm 2.8$ & $6.3 \pm 2.2$ & $5.9 \pm 1.8$ & $4.2 \pm 2.7$ & $7.9 \pm 1.2$ & $7.4 \pm 2.5$ & $7.7 \pm 1.4$ \\
\hline Post 1 & $3.2 \pm 3.3$ & $7.2 \pm 1.6^{*}$ & $8.2 \pm 1.0$ & $7.7 \pm 1.1^{* *}$ & $6.2 \pm 2.7$ & $8.0 \pm 0.8$ & $8.5 \pm 1.4$ & $8.3 \pm 0.7^{\star}$ \\
\hline Post 2 & $2.7 \pm 3.6$ & $4.1 \pm 2.9$ & $8.1 \pm 1.0^{*}$ & $6.1 \pm 1.7$ & $4.3 \pm 3.2$ & $5.6 \pm 3.2^{*}$ & $8.5 \pm 1.1$ & $7.0 \pm 2.1^{*}$ \\
\hline
\end{tabular}


For $M$. trapezius at the trot, there was a statistically significant improvement in the E-score only for the left-side between the Pre and Post 2 measurements (P $<0.01)$.

For M. latissimus dorsi at the walk, there was a statistically significant improvement in the E-score for the left-side between the Pre and Post 1 measurement only $(\mathrm{P}<0.05)$, and for the $\mathrm{E}$ and $\mathrm{T}$-scores for the right-side between the Pre and Post $1(\mathrm{P}<0.05)$ yet an impaired E, S, T and ST-score for the Pre vs Post 2 measurements $(\mathrm{P}<0.05)$.

For M. latissimus dorsi at the trot, there was a statistically significant improvement in the S and ST-scores between Pre and Post1 measurements for the left-side $(\mathrm{P}<0.05$ and $\mathrm{P}<0.01)$ and an improvement in the $\mathrm{T}$-score between the Pre and Post 2 measurement only $(P<0.05)$. For the right side, a statistically significant improvement in the ST-score $(\mathrm{P}<0.05)$ was found between the Pre and Post 1 measurements, and for the $S$ and ST-scores an impaired $S$ and ST-score for the Pre vs Post 2 measurements $(\mathrm{P}<0.05)$.

The balance scores for $M$. trapezius at the walk were $0.5 \pm 7.0$ Pre and $1.7 \pm$ 9.2 and $1.6 \pm 4.2$ for Post 1 and Post 2, respectively. For M. trapezius at the trot the balance scores were 2.8 \pm 3.1 Pre and $2.9 \pm 6.5$ and $1.4 \pm 6.5$ for Post 1 and Post 2, respectively.

The balance scores for $M$. latissimus dorsi at the walk were $2.8 \pm 6.2$ Pre and $1.6 \pm 4.2$ and $0.5 \pm 9.8$ for Post 1 and Post 2, respectively. For M. latissimus dorsi at the trot the balance scores were $6.6 \pm 7.7$ Pre and $3.4 \pm 5.4$ and $2.9 \pm 7.7$ for Post 1 and Post 2, respectively.

The mfBIA findings lend support to the AMG data for the shoulder muscle $M$. trapezius at the walk, where not only does the E- and T-score improve significantly with treatment, but also the ST-score on the right-hand side significantly improves, indicating more force production, complying with the significant increase in the PA parameter for this muscle and side of the horses. At the trot, this muscle group was found to work very evenly (right vs left side) with regard to the acoustic myography parameters.

\section{Discussion}

This study is the first to examine the effect of low-frequency ultrasound therapeutic treatment on the $M$. trapezius and M. latissimus dorsi of horses, assessing any changes in static as well as dynamic muscle properties.

The mfBIA data for the shoulder muscle $M$. trapezius reveals a significant improvement in the Mc and PA parameters for the right-hand side of the horses after treatment compared with the pre-treatment values. Membrane capacitance (Mc) defines the status of the muscle fibre membrane potential, and as such it illustrates a combination of fibre health and transport status of the membrane. However, it is also very much related to the thickness of the cell membrane [11] [12] [13]. The phase angle (PA) has been used as a means of assessing changes in an individual's body mass, representing as it does a ratio of R and Xc [11] [14]. 
What the EQ Pro ultrasound therapy indicates, therefore, is an improvement in not only the mass of the right-hand side shoulder muscles but also its cellular health. In fact, the results show that the right-hand side M. trapezius has a lower Mc and PA at the start of the study and becomes more even after EQ Pro Therapy treatment.

For M. latissimus dorsi, at the walk there was an initial imbalance in the acoustic myography signals such that the left-hand side appeared to work more (lower scores), but this imbalance was resolved with EQ Pro Therapy ultrasound treatment such that both sides worked with very similar scores. A similar finding of an imbalance in the AMG scores was found pre-treatment for this muscle at the trot, and after treatment a more balanced set of scores was measured, with the right-hand side engaging and working more than it did pre-treatment.

Similar changes in mfBIA values have been documented after treatment of another kind (myofascial release), with some myofascial groups improving immediately with treatment, and others worsening temporarily, before also finally improving after a number of hours (24 - 48) [15]. Indeed, this form of compensation over time to treatment reported by Elbrønd and colleagues [15] is a widely known phenomenon. Likewise, in the present study, the data suggest that for the horses measured there was a tendency based on the pre-treatment values for the horses to slightly overuse their left-hand side M. trapezius and M. latissimus dorsi muscles at the walk and trot.

In terms of limitations, this study should be seen as a preliminary investigation as there are relatively few horses measured, and they were not recruited with identical, or indeed symmetrically diagnosed symptoms. Another limitation might also be a lack of a control group for this study. Horses were selected based on them having muscles that were mildly painful to palpation and atrophied. There is now a need, therefore, for a more carefully planned study to confirm and further investigate these interesting findings. With regards to the AMG data, which show a degree of variation, a great deal of this is most likely associated with treatment-related compensation, as explained earlier [15], particularly since such compensation changes are very much related to the underlying issues of the individual subjects. However, the role, if any, of massage as a result of treatment, cannot be ignored as a contributing factor in this case. Yet despite all of these issues, this study indicates that with EQ Pro Therapy ultrasound treatment the initial muscle imbalance disappeared, both in terms of the static mfBIA measurements and also in terms of the dynamic acoustic myography recordings. Furthermore, since Post 2 measurements were taken 5 months and 10 days after treatment ended, one might suggest that EQ Pro Therapy ultrasound effects are not only temporary and analgesic, but of a longer duration.

\section{Conclusion}

It is concluded that EQ Pro Therapy ultrasound treatment appears to have a lasting beneficial effect on soft tissue shoulder muscles both in terms of their 
mfBIA parameters and their dynamic AMG measurements. These preliminary results suggest improvements in muscle mass, cellular health, and improved force production, better coordination, and improved lateral balance, although a more detailed study is now needed. EQ Pro Therapy ultrasound treatment may be a promising means of treating injured shoulder muscles in horses.

\section{Acknowledgments}

The authors would like to thank Caroline Hegarty and Equitopia CA for use of their facilities, and the horse owners for their generous help.

\section{Authors' Contributions}

Project design was arrived at by DI and AH. Treatment was performed by SL, whilst data collection was performed by DI and SL, and analysis was performed blinded by AH. All authors contributed to the interpretation and writing of this manuscript.

\section{Conflicts of Interest}

$\mathrm{AH}$ is in the process of establishing a company to produce and market the Acoustic Myography equipment (CURO-Diagnostics ApS), but at no time was he involved in collecting the data. Neither author has any financial interest or otherwise in the EQ Pro Therapy device, nor did they receive payment for this study.

\section{References}

[1] Jönsson, L., Roepstorff, L., Egenvall, A., Näsholm, A., Dalin, G. and Philipsson, J. (2013) Prevalence of Clinical Findings at Examinations of Young Swedish Warmblood Riding Horses. Acta Veterinaria Scandinavica, 55, Article No. 34. https://doi.org/10.1186/1751-0147-55-34 http://www.actavetscand.com/content/55/1/34

[2] Wilk, I., Wnuk-Pawlak, E., Janczarek, I., Kaczmarek, B., Dybczynska, M. and Przetacznik, M. (2020) Distribution of Superficial Body Temperature in Horses Ridden by Two Riders with Varied Body Weights. Animals, 10, Article No. 340. https://doi.org/10.3390/ani10020340

[3] Clayton, H.M., Hampson, A., Fraser, P., White, A. and Egenvall, A. (2018) Comparison of Rider Stability in a Flapless Saddle Versus a Conventional Saddle. PLoS ONE, 13, e0196960. https://doi.org/10.1371/journal.pone.0196960

[4] Penham, C., Licka, T., Schobesberger, H. and Meschan, E. (2004) Influence of the Rider on the Variability of the Equine Gait. Human Movement Science, 23, 663-671.

[5] Adair, H.S. and Levine, D. (2019) Effects of 1-MHz Ultrasound on Epaxial Muscle Temperature in Horses. Frontiers in Veterinary Science, 6, Article 177. https://doi.org/10.3389/fvets.2019.00177

[6] Carrozzo, U., Toniato, M. and Harrison, A. (2019) Assessment of Noninvasive Low-Frequency Ultrasound as Means of Treating Injuries to Suspensory Ligaments in Horses: A Research Paper. Journal of Equine Veterinary Science, 80, 80-89.

[7] Harrison, A.P., Elbrønd, V.S., Riis-Olesen, K. and Bartels, E.M. (2015) Multi-Frequency 
Bioimpedance in Equine Muscle Assessment. Physiological Measurement, 36, 453-464. https://doi.org/10.1088/0967-3334/36/3/453

[8] Harrison, A.P. (2018) A More Precise, Repeatable and Diagnostic Alternative to Surface Electromyography-An Appraisal of the Clinical Utility of Acoustic Myography. Clinical Physiology and Functional Imaging, 38, 312-325. https://doi.org/10.1111/cpf.12417

[9] Dyson, S., Berger, J., Ellis, A.D. and Mullard, J. (2018) Development of an Ethogram for a Pain Scoring System in Ridden Horses and Its Application to Determine the Presence of Musculoskeletal Pain. Journal of Veterinary Behavior, 23, 47-57.

[10] Claudel, C.G., Ahmed, W., Elbrond, V.S., Harrison, A.P. and Bartels, E.M. (2018) The Relation between Maximal Voluntary Force in M. Palmaris Longus and the Temporal and Spatial Summation of Muscle Fiber Recruitment in Human Subjects. Physiological Reports, 6, e13580. https://doi.org/10.14814/phy2.13580

[11] Ivorra, A. (2003) Bioimpedance Monitoring for Physicians: An Overview. Centre National de Microelectronica, Barcelona.

[12] Dodde, R.E., Bull, J.L. and Shih, A.J. (2012) Bioimpedance of Soft Tissue under Compression. Physiological Measurement, 33, 1095-1109.

[13] Tan, Q., Ferrier, G.A., Chen, B.K., Wang, C. and Sun, Y. (2012) Quantification of the Specific Membrane Capacitance of Single Cells Using a Microfluidic Device and Impedance Spectroscopy Measurement. Biomicrofluidics, 6, Article ID: 034112.

[14] Bartels, E.M., Sorensen, E.R. and Harrison, A.P. (2015) Multi-Frequency Bioimpedance in Human Muscle Assessment. Physiological Reports, 3, e12354. https://doi.org/10.14814/phy2.12354

[15] Elbrønd, V.S., Krasnodebska, M.J. and Harrison, A. (2015) Multi-Frequency Bioimpedance and Myofascial Release Therapy: An Equine "AtlasOrange1" Validation Study. Medical Research Archives, 3, e12345.

https://esmed.org/MRA/mra/article/view/124. 